\title{
Prevalencia y evolución de síntomas depresivos en pacientes hospitalizados por infarto agudo al miocardio y su relación con procedimientos de revascularización
}

\author{
JORGE CALDERÓN ${ }^{1}$, LUIGI GABRIELLI², MATÍAS GONZÁLEZ ${ }^{1}$, \\ LUIS VILLARROEL ${ }^{3}$, PABLO CASTRO ${ }^{2}$, RAMÓN CORBALÁN ${ }^{2}$
}

\section{Depressive symptoms among patients with acute myocardial infarction. Relationship with revascularization therapy}

Background: Persistence of depressive symptoms after myocardial infarction (MI) is associated with an adverse outcome. The relationship between depression and Invasive Revascularization Therapy (IRT) is not yet fully understood. Aim: To compare the frequency of depressive symptoms and other psychosocial variables among patients with MI, undergoing or not undergoing IRT. Material and Methods: Prospective evaluation of 45 patients aged $58 \pm 11$ years ( $87 \%$ males) with a diagnosis of MI (Killip I and II). On admission to hospital and at follow up 3 months after discharge, all patients completed the Beck Depression Inventory (BDI), the Zung Anxiety Inventory (ASI), and the Medical Outcomes Study (MOS) social support survey. Depressive symptoms were considered to be present if the BDI score was over 10. Results: Thirty seven percent were hypertensive, $15 \%$ diabetic and $44 \%$ smokers. Sixty four percent of patients underwent IRT (11.1\% revascularization surgery and $53.3 \%$ coronary angioplasty). Forty four percent of patients scored over 10 in the BDI at baseline assessment and $26.5 \%$ at 3 months follow-up $(p<0.01)$. At baseline BDI score was $10.2 \pm 5.1$ and $9.1 \pm 4.4$ among patients subjected or not subjected to IRT, respectively (NS). The figures at 3 months of follow up were $9.9 \pm 5.6$ and $4.1 \pm$ 2.5 , respectively $(p<0,01)$. At baseline and three months BDI, anxiety and perceived social support were significantly correlated. Conclusions: Depressive symptoms were frequent after MI in this group of patients, and decreased at follow up only among patients not subjected to IRT.

(Rev Med Chile 2010; 138: 701-706).

Key words: Depression; Myocardial infarction; Myocardial revascularization.

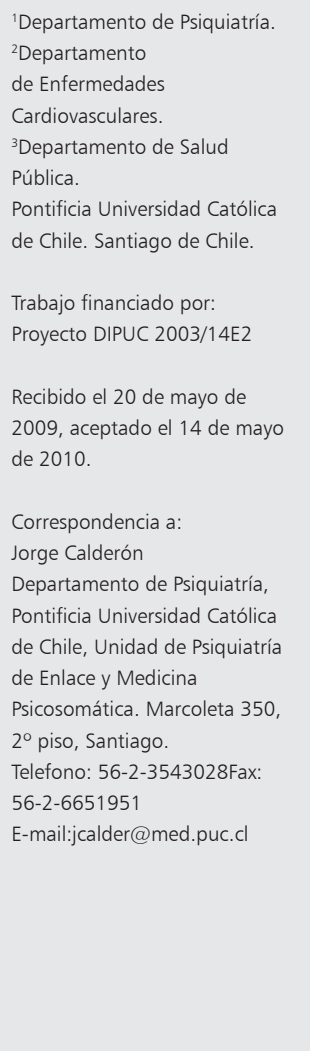

L a depresión es una patología frecuente y con un impacto significativo en la funcionalidad y la salud general de la población mundial. Se estima que su prevalencia en el mundo oscila entre $12 \%$ y $17 \%{ }^{1}$. Estudios poblacionales en Chile arrojan cifras similares ${ }^{2,3}$.

La prevalencia de depresión en el hospital general es aun más significativa, con una prevalencia puntual entre $15 \%$ y $36 \%$ considerando todos los trastornos depresivos ${ }^{4,5}$. Aproximadamente entre 15 y $25 \%$ de los pacientes hospitalizados por IAM (Infarto agudo al miocardio) sufren de depresión mayor ${ }^{6,7}$, y la tasa de mortalidad en este grupo es unas 2 veces mayor que en los pacientes sin depresión $^{8}$. En Chile, el único estudio de prevalencia de síntomas depresivos en pacientes con antecedente 
de infarto al miocardio, arrojó una prevalencia de $38,3 \%$.

La depresión no parece ser un mero marcador de indicadores clínicos que predicen mortalidad post IAM, como lo demuestra un estudio reciente en el que se analiza la relación de depresión y un índice de predicción clínico de mortalidad. En este estudio, no hubo relación entre síntomas depresivos y el índice de predicción. Los síntomas depresivos se relacionaron con mayor mortalidad, incluso después de controlar las variables de índice de predicción y disfunción ventricular izquierda, poniendo de manifiesto la posibilidad de una relación única entre depresión y pronóstico post IAM independiente de las variables de riesgo habituales, y su severidad ${ }^{10}$.

Diversos mecanismos fisiopatológicos podrían explicar la relación entre depresión y enfermedad coronaria: efecto sobre la perfusión miocardica, desregulación autonómica, activación plaquetaria, actividad del eje hipotalámico-hipofisiarioadrenal, y procesos inflamatorios dentro de los más estudiados ${ }^{11}$, haciendo que la posible relación entre depresión y enfermedad coronaria sea de carácter complejo.

En un estudio transversal de más de 2.000 pacientes, que usó un modelo de estrés psicológico compuesto que incluye depresión, ansiedad, fatiga y dificultades para dormir, Wolff y colaboradores demostraron una relación lineal con la posibilidad de presencia de placa ateromatosa carotidea, que fue independiente de otros factores de riesgo ${ }^{12}$.

Los síntomas depresivos post IAM tienden a desaparecer en alrededor de 50\% de los casos en el seguimiento a mediano plazo, sugiriendo un carácter transitorio y reactivo al IAM. La persistencia de los síntomas depresivos por un tiempo más prolongado tendría, en contraste, mayor significación, en su relación con el pronóstico cardiovascular ${ }^{13}$.

Por otra parte, la presencia de síntomas depresivos al momento de la evaluación angiográfica parece asociarse a la necesidad de revascularización y los costos totales durante el año siguiente. Sullivan y colaboradores comunicaron que los pacientes con síntomas depresivos más severos, fueron más frecuentemente sometidos a angioplastia, en comparación con los no deprimidos ${ }^{14}$.

En suma, la depresión parece ser un síntoma frecuente en pacientes con IAM, y constituir un factor de riesgo cardiovascular de importancia, particularmente cuando se mantiene en el tiempo, y no sólo un marcador de los factores de riesgo habituales.

El presente trabajo tiene como objetivo describir la presencia y evolución de los síntomas depresivos y otras variables psicosociales en pacientes con IAM, y su relación con procedimientos de revascularización. Nuestra hipótesis es que los síntomas depresivos serán frecuentes y tendrán un carácter más persistente en los pacientes sometidos a procedimiento de revascularización, dando cuenta de un grupo más vulnerable en relación a la asociación entre depresión y pronóstico en pacientes con IAM.

\section{Material y Métodos}

\section{Pacientes}

Cohorte prospectiva de pacientes ingresados a la unidad coronaria del Hospital Clínico de la Pontificia Universidad Católica de Chile, con diagnóstico de IAM, durante los años 2005 y 2006. Los criterios de inclusión fueron: pacientes ingresados con diagnóstico de IAM basado en criterios clínicos (dolor retroesternal mayor de 20 minutos en reposo), electrocardiográficos (infradesnivel del ST mayor de 0,5 $\mathrm{mm}$ en dos derivadas contiguas o inversión de onda $\mathrm{T}$ ), y enzimáticos (troponina I > $0,05 \mathrm{ng} / \mathrm{mL}$ ), mayores de 21 años, Killip I y II, que firmaron un consentimiento informado aprobado por el Comité de Ética de nuestra institución. Los criterios de exclusión fueron: la presencia de una neoplasia, IAM con supradesnivel del ST, shock cardiogénico, complicaciones mecánicas post infarto, enfermedad asociada severa, uso de cocaína, imposibilidad de seguir controles, imposibilidad de responder a los cuestionarios.

De un total de 32 pacientes (71\%) que fueron sometidos a coronariografía diagnóstica, 29 $(64,4 \%)$ recibieron TRI (terapia de revascularización invasiva). De estos, 5 pacientes (11\%) fueron sometidos a cirugía de revascularización miocárdica (CRM), y $24(53,3 \%)$ a angioplastia coronaria.

La indicación de estudio invasivo estuvo definida por estratificación de riesgo según TIMI score para IAM sin supradesnivel del ST con 4 o más puntos y la indicación de revascularización se hizo según el hallazgo de lesiones coronarias significativas en aquellos pacientes estudiados según criterio del hemodinamista y cardiólogo clínico. 
Depresión post infarto cardíaco y revascularización - J. Calderón et al

La decisión de cirugía o angioplastia estuvo dada por: enfermedad de múltiple vaso, complejidad de lesión y presencia de disfunción ventricular.

\section{Instrumentos de investigación}

Todos los pacientes que ingresaron al estudio fueron evaluados inmediatamente antes del alta o a los 5 días post IAM, y a los 3 meses post alta, completando: una ficha sociodemográfica en la que se incluyeron las variables como edad, sexo, escolaridad, estado civil, etc; una ficha médica que incluyó factores de riesgo para enfermedad cardiovascular y los exámenes de laboratorio apropiados, además de los antecedentes psiquiátricos de relevancia.

Instrumentos psicométricos: Escala de Red Social MOS, Cuestionario autoadministrado de 20 ítems, que valora apoyo social tanto estructural como percibido ${ }^{15}$; (Zung Anxiety inventory (ASI), que es un instrumento de screening ampliamente usado para la detección de ansiedad ${ }^{16}$; Anger-out Expression Scale de Spielberger, que es un instrumento que permite medir expresión de rabia como estilo caracterológico, la que se ha vinculado a mayor incidencia de nuevos eventos coronarios en pacientes con antecedentes de $\mathrm{IAM}^{17}$; Beck Depression Inventory (BDI), que es un instrumento de screening orientado a detectar síntomas depresivos, usado ampliamente para la investigación de trastornos afectivos y particularmente útil en investigación de trastornos afectivos en pacientes con patología médica. Se caracteriza por ser de fácil administración, autoaplicación, e interpretación ${ }^{18}$. En el presente trabajo, el puntaje de corte para el BDI fue de 10 puntos, que es el puntaje de corte estándar. El BDI es probablemente el instrumento de depresión más frecuentemente utilizado en los estudios de depresión e IAM, y tiene una sensibilidad y especificidad similar a los estudios con este instrumento en la atención primaria, que es de $85 \%$ y $74 \%$ respectivamente ${ }^{19}$. El efecto de la depresión sobre el pronóstico del IAM, tiene una gradiente de intensidad, con un aumento proporcional de la morbimortalidad cardiovascular en relación a la severidad de los síntomas depresivos ${ }^{7}$, lo que hace recomendable incluir toda la gama de severidad de los síntomas depresivos. Por otro lado, en diversos estudios de prevalencia de depresión en pacientes con IAM, la definición de caso de depresión no parece modifi- carse significativamente cuando se compara una entrevista semiestrucuturada, con un instrumento de autoevaluación de síntomas depresivos ${ }^{8,20}$.

Todos estos instrumentos cuentan con validación al español. El BDI ha sido utilizado en varios estudios en Chile, algunos de ellos en pacientes con patología médica ${ }^{9,21,22}$.

En el presente trabajo se estableció una correlación de los puntajes de los diversos instrumentos como variables continuas, por lo que no se usó puntaje de corte, con la excepción del BDI para efectos de determinar la prevalencia de síntomas depresivos en nuestra muestra.

\section{Estadística}

Las variables se expresaron como porcentaje o promedios $\pm \mathrm{DE}$ según corresponda. Se utilizaron Mann Whitney U test y test de chi-cuadrado para comparación de variables. Para comparar la evolución de síntomas depresivos según grupo se utilizó test de la $t$ de Student para muestras pareadas. Para la evaluación de correlaciones entre distintas variables se utilizó el método de Pearson.

\section{Resultados}

La edad promedio de la muestra corresponde a 58 años y $87 \%$ corresponde a pacientes de sexo masculino. Las características clínicas basales de la muestra se describen en la Tabla 1.

La prevalencia de síntomas depresivos en la medición basal fue de 44,4\% (con puntaje en el BDI $>$ a 10), disminuyendo a $26,5 \%$ a los 3 meses $(\mathrm{p}<0,01)$. Este puntaje de corte en el BDI define el grupo de pacientes con síntomas depresivos "clínicamente significativos" (Figura 1).

Tabla 1. Características clínicas basales de pacientes con IAM

\begin{tabular}{|ll|}
\hline $\mathbf{n}$ & $\mathbf{4 5}$ \\
\hline Edad (años \pm DE) & $58 \pm 11$ \\
\hline Género masculino (\%) & 87 \\
\hline Hipertensión (\%) & 37 \\
\hline Diabetes (\%) & 15 \\
\hline Tabaquismo (\%) & 44 \\
\hline Dislipidemia (\%) & 49 \\
\hline IAM previo (\%) & 11 \\
\hline
\end{tabular}




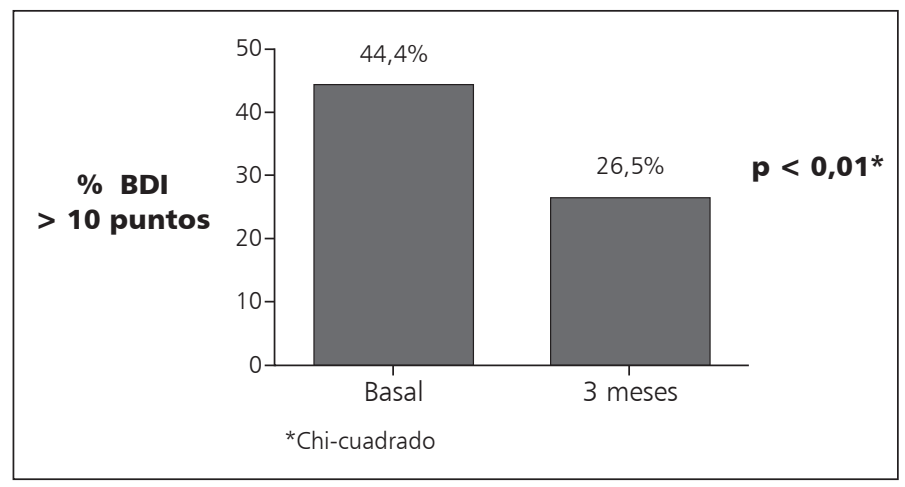

Figura 1. Prevalencia de síntomas depresivos en pacientes con IAM, durante hospitalización (basal) y al seguimiento (3 meses).

Tabla 2. Características comparativas entre grupo de pacientes con IAM con síntomas depresivos clínicamente significativos (BDI > 10) versus no depresivos $(\mathrm{BDI}<10)$

\begin{tabular}{|lccc|}
\hline & $\begin{array}{c}\text { BDI < 10 } \\
(\mathbf{n}=\mathbf{2 4})\end{array}$ & $\begin{array}{c}\text { BDI > 10 } \\
(\mathbf{n = 2 1 )}\end{array}$ & Valor $\mathbf{p}$ \\
\hline Hombres (\%) & 95,8 & 76,2 & NS \\
\hline Edad (años \pm DE) & $58,6 \pm 11,3$ & $57,6 \pm 12,2$ & NS \\
\hline Tabaquismo (\%) & 57,1 & 42,9 & NS \\
\hline DM 2 (\%) & 29,1 & 28,6 & NS \\
\hline HTA (\%) & 58,8 & 41,2 & NS \\
\hline Dislipidemia (\%) & 44,4 & 55,6 & NS \\
\hline IAM previo (\%) & 16,7 & 4,8 & NS \\
\hline
\end{tabular}

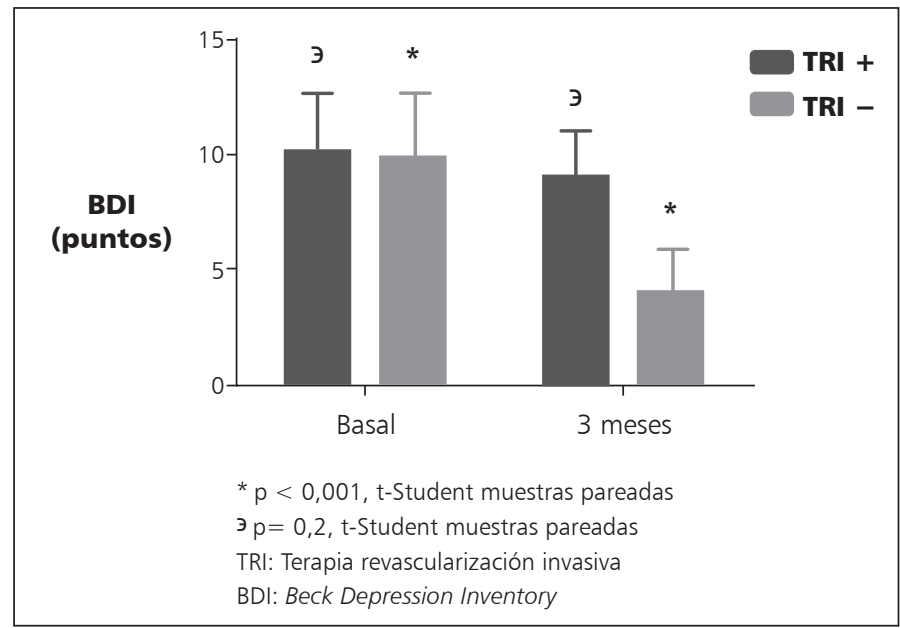

Figura 2. Evolución de síntomas depresivos en pacientes con IAM en relación a realización de procedimiento de revascularización (TRI).
Como se demuestra en la Tabla 2, no hubo diferencias estadísticamente significativas al comparar los grupos con síntomas depresivos versus los que no tienen estos síntomas, en relación a sexo, edad, tabaquismo, diabetes mellitus, hipertensión arterial, dislipidemia y antecedente de IAM previo.

$\mathrm{Al}$ analizar los síntomas depresivos en relación a TRI, los puntajes del BDI fueron similares en ambos grupos en la medición basal. Sin embargo, esta relación se perdió a los 3 meses, estableciéndose una diferencia estadísticamente significativa entre los 2 grupos, con una persistencia de síntomas depresivos en los pacientes sometidos a TRI $(\mathrm{p}<0,2)$ versus una disminución de estos síntomas en los pacientes no sometidos a TRI $(\mathrm{p}<$ 0,001) (Figura 2).

Los síntomas ansiosos se correlacionaron positivamente con los depresivos ( $\mathrm{p}<0,002)$, en tanto los síntomas depresivos se correlacionaron negativamente con los niveles de apoyo social percibido. No hubo, sin embargo, una relación entre síntomas depresivos y hostilidad.

\section{Discusión}

Nuestro estudio representa el primer trabajo en nuestro medio en el que se realiza un seguimiento de pacientes con infarto agudo al miocardio, con el objetivo de analizar su relación con diversas variables psicosociales en el tiempo, y los procedimientos de revascularización realizados.

La prevalencia de síntomas depresivos clínicamente significativos fue de $44,4 \%$, durante el período de hospitalización, ligeramente más alto que la prevalencia encontrada en un trabajo previo realizado en un hospital de Santiago, el que reportó una prevalencia de $38,3 \%$, aunque 
el puntaje de corte en esta medición fue más alto que el habitual, captando un grupo de pacientes más severos. Este último es un estudio de prevalencia de sintomatología depresiva en pacientes con diagnóstico de IAM, por lo que no incluye información acerca del seguimiento y la evolución de síntomas depresivos?.

En concordancia con otros estudios realizados, en nuestro estudio los síntomas depresivos disminuyeron significativamente al control de los 3 meses, apoyando la noción del carácter transitorio de un grupo importante de síntomas depresivos post IAM. Es interesante notar, sin embargo, que esta disminución de síntomas depresivos al seguimiento se produjo a expensas de los pacientes que no fueron sometidos a revascularización. De hecho, no hubo una disminución significativa de los síntomas depresivos en los pacientes que si fueron sometidos a revascularización, confirmando nuestra hipótesis inicial.

La persistencia de síntomas depresivos en pacientes sometidos a TRI plantea interrogantes que son interesantes. Es improbable que esto se deba a diferencias significativas en la repercusión funcional del IAM, puesto que en los criterios de inclusión sólo se consideraron pacientes con repercusión hemodinámica leve o moderada. Sería, por otro lado orientador estudiar el efecto de la TRI sobre la percepción subjetiva del paciente acerca de su pronóstico cardiovascular, pues esto podría relacionarse indirectamente con la persistencia de síntomas depresivos, en el caso de una apreciación negativa. Los estudios, sin embargo, sugieren una independencia de la variable depresión y la severidad del $\mathrm{IAM}^{23}$.

La asociación entre depresión e infarto es un hecho firmemente establecido en la actualidad, sin embargo, los 2 estudios multicéntricos realizados al presente, que evalúan el efecto del tratamiento de la depresión sobre el pronóstico cardiovascular, no han mostrado que éste sea significativo ${ }^{24}$. De los análisis secundarios de estos estudios publicados recientemente, se desprende sin embargo, la observación de que los pacientes infartados refractarios a tratamiento antidepresivo, parecen tener un peor pronóstico cardiovascular y que este hecho podría estar asociado al polimorfismo del gen transportador de serotonina ${ }^{25,26}$.

Nuestro trabajo plantea la posibilidad que la interacción compleja entre depresión e IAM y enfermedad coronaria, pueda estar expresándose en la persistencia de los síntomas depresivos en pacientes con enfermedad coronaria más severa.

La presencia simultánea de factores psicosociales (depresión, ansiedad, apoyo social percibido) parece tener un efecto sinérgico y negativo sobre el pronóstico cardiovascular ${ }^{27}$. En nuestro estudio, tanto la ansiedad como el apoyo social percibido, tuvieron una relación significativa con los síntomas depresivos.

En una publicación reciente, Sheps y colaboradores estiman que al menos el $26 \%$ de las muertes por IAM podría ser atribuido a isquemia inducida por estrés ${ }^{28}$. La importancia epidemiológica que se deriva de esta observación hace perentorio avanzar en la descripción más detallada de los subgrupos más vulnerables.

La relación inversa entre depresión y apoyo social percibido, establecida en nuestro estudio, plantea la necesidad de incorporar esta última variable como parte del screening habitual de los pacientes cardiológicos. En un estudio de seguimiento post-quirúrgico tras el diagnóstico de IAM, Pirraglia et al, identificaron al apoyo social deficiente, entre otros, como un factor predictivo de depresión ${ }^{29}$.

En suma, el presente trabajo demuestra una alta prevalencia de síntomas depresivos en pacientes ingresados a una unidad coronaria con diagnóstico de IAM. Estos síntomas depresivos tienden a mantenerse en los pacientes que fueron sometidos a TRI, hecho que podría estar señalando la presencia de un subgrupo más vulnerable a la interacción entre depresión y otros factores psicosociales y el pronóstico cardiovascular.

\section{Referencias}

1. Honig A, Praag HMV. Depression: neurobiological, psychopathological, and therapeutic advances. Chichester: Wiley; 1997.

2. Araya R, Rojas G, Fritsch R, Acuna J, Lewis G. Common mental disorders in Santiago, Chile: prevalence and socio-demographic correlates. Br J Psychiatry 2001; 178: 228-33.

3. Vicente B, Kohn R, Rioseco P, Saldivia S, Levav I, Torres S. Lifetime and 12-month prevalence of DSM-III-R disorders in the Chile psychiatric prevalence study. Am J Psychiatry 2006; 163: 1362-70.

4. Magni G, Schifano F, De Leo D. Assessment of depression in an elderly medical population. J Affect Disord 1986; 11: 121-4. 
5. Feldman E, Mayou R, Hawton K, Ardern M, Smith EB. Psychiatric disorder in medical in-patients. Q J Med 1987; 63: 405-12.

6. Barefoot JC, Helms MJ, Mark DB, et al. Depression and long-term mortality risk in patients with coronary artery disease. Am J Cardiol 1996; 78: 613-7.

7. Frasure-Smith N, Lesperance F, Talajic M. Depression and 18-month prognosis after myocardial infarction. Circulation 1995; 91: 999-1005.

8. Van Melle JP, De Jonge P, Spijkerman TA, et al. Prognostic association of depression following myocardial infarction with mortality and cardiovascular events: a meta-analysis. Psychosom Med 2004; 66: 814-22.

9. Blumel B, Gibbons A, Kanacri A, Kerrigan N, Florenzano R. [Depressive symptoms after an acute myocardial infarction]. Rev Med Chile 2005; 133: 1021-7.

10. Kronish IM, Rieckmann N, Schwartz JE, Schwartz DR, Davidson KW. Is depression after an acute coronary syndrome simply a marker of known prognostic factors for mortality? Psychosom Med 2009; 71: 697-703.

11. Musselman DL, Evans DL, Nemeroff CB. The relationship of depression to cardiovascular disease: epidemiology, biology, and treatment. Arch Gen Psychiatry 1998; 55: 580-92.

12. Wolff B, Grabe HJ, Volzke H, et al. Relation between psychological strain and carotid atherosclerosis in a general population. Heart 2005; 91: 460-4.

13. Glassman AH, O'connor CM, Califf RM, et al. Sertraline treatment of major depression in patients with acute MI or unstable angina. JAMA 2002;288:701-9.

14. Sullivan MD, Lacroix AZ, Spertus JA, Hecht J, Russo J. Depression predicts revascularization procedures for 5 years after coronary angiography. Psychosom Med 2003; 65: 229-36.

15. Sherbourne CD, Stewart AL. The MOS social support survey. Soc Sci Med 1991; 32: 705-14.

16. Zung WW. A rating instrument for anxiety disorders. Psychosomatics 1971; 12: 371-9.

17. Spielberger CJG, Russel S, Crane R. Assessment of Anger: The State-Trait Anger Scale In: Butcher J SC, ed. Advances in Personality Assessment. Hillsdale, NJ: Lawrence Erlbaum Associates; 1983.

18. Beck AT, Ward CH, Mendelson M, Mock J, Erbaugh J. An inventory for measuring depression. Arch Gen Psychia- try 1961; 4: 561-71.

19. Thombs BD, Magyar-Russell G, Bass EB, et al. Performance characteristics of depression screening instruments in survivors of acute myocardial infarction: review of the evidence. Psychosomatics 2007; 48: 185 94.

20. Barth J, Schumacher M, Herrmann-Lingen C. Depression as a risk factor for mortality in patients with coronary heart disease: a meta-analysis. Psychosom Med 2004; 66: 802-13.

21. Heerlein A, Gabler G, Chaparro C, Kraus A, Richter P, Berkau C. [Cross cultural psychometric comparison of major depression in Chile and Germany]. Rev Med Chile 2000; 128: 613-8.

22. Dois CA, Cazenave A. [Frequency of depression among hypertensive subjects in a primary care clinic]. Rev Med Chile 2009; 137: 475-80.

23. Burg MM, Benedetto MC, Rosenberg R, Soufer R. Presurgical depression predicts medical morbidity 6 months after coronary artery bypass graft surgery. Psychosom Med 2003; 65: 111-8.

24. Carney RM, Freedland KE, Sheps DS. Depression is a risk factor for mortality in coronary heart disease. Psychosom Med 2004; 66: 799-801.

25. De Jonge P, Honig A, Van Melle JP, et al. Nonresponse to treatment for depression following myocardial infarction: association with subsequent cardiac events. Am J Psychiatry 2007; 164: 1371-8.

26. Otte C, Mccaffery J, Ali S, Whooley MA. Association of a serotonin transporter polymorphism (5-HTTLPR) with depression, perceived stress, and norepinephrine in patients with coronary disease: the Heart and Soul Study. Am J Psychiatry 2007; 164: 1379-84.

27. Rozanski A, Blumenthal JA, Kaplan J. Impact of psychological factors on the pathogenesis of cardiovascular disease and implications for therapy. Circulation 1999; 99: 2192-217.

28. Sheps DS, Soufer R, Freedland KE. Psychological stress and myocardial ischemia: understanding the link and the implications. Psychosom Med 2007; 69: 491-2.

29. Pirraglia PA, Peterson JC, Williams-Russo P, Gorkin L, Charlson ME. Depressive symptomatology in coronary artery bypass graft surgery patients. Int J Geriatr Psychiatry 1999; 14: 668-80. 